\title{
Typologies of student experiences and constructed meanings of learning in international placements
}

*Sara Ashencaen Crabtree, Jonathan Parker, Azlinda Azman, Dolly Paul Carlo

*Correspondent address

Dr Sara Ashencaen Crabtree

Bournemouth University

School of Health \& Social Care

$2^{\text {nd }}$ Floor Royal London House,

Christchurch Road, Bournemouth, Dorset UK BH1 3LT

scrabtree@bournemouth.ac.uk 


\begin{abstract}
This paper discusses findings from a three-year British Council funded project into social work student placements in Malaysia. Processes of student learning in unfamiliar cultural contexts were examined in relation to three cohorts of students. Here typologies of experience influencing learning, elicited from student-recorded data, are discussed. Analysis suggests that students undergo a process of liminality, adjustment or resistance to the contexts of community, culture and placements encountered in international settings. Emergent themes were identified as naïve acceptance, critical revelation, critical observation, epiphany, critical reactionary, professional rejection and antagonistic response. Implications for international placements are examined based on the data.
\end{abstract}

Keywords: practice learning, cultural competency, international 